\title{
Injection of genetically engineered fibroblasts corrects regenerated human epidermolysis bullosa skin tissue
}

\author{
Susana Ortiz-Urda, ${ }^{1}$ Qun Lin,${ }^{1}$ Cheryl L. Green, ${ }^{1}$ Douglas R. Keene, ${ }^{2}$ \\ M. Peter Marinkovich, ${ }^{1}$ and Paul A. Khavari ${ }^{1}$ \\ ${ }^{1}$ Veterans Affairs Palo Alto Healthcare System, Palo Alto, California, USA, and the Program in Epithelial Biology, \\ Stanford University School of Medicine, Stanford, California, USA \\ ${ }^{2}$ Shriners Hospital for Children, Portland, Oregon, USA
}

Current therapeutic strategies for genetic skin disorders rely on the complex process of grafting genetically engineered tissue to recipient wound beds. Because fibroblasts synthesize and secrete extracellular matrix, we explored their utility in recessive dystrophic epidermolysis bullosa (RDEB), a blistering disease due to defective extracellular type VII collagen. Intradermal injection of RDEB fibroblasts overexpressing type VII collagen into intact RDEB skin stably restored correctly localized type VII collagen expression in vivo and normalized hallmark RDEB disease features, including subepidermal blistering and anchoring fibril defects. Fibroblast injection thus provides a simplified approach to correcting human disorders of secreted matrix proteins.

This article was published online in advance of the print edition. The date of publication is available

from the JCI website, http://www.jci.org. J. Clin. Invest. 111:251-255 (2003). doi:10.1172/JCI200317193.

\section{Introduction}

Recessive dystrophic epidermolysis bullosa (RDEB) is among the numerous human skin diseases that involve secreted matrix proteins. Initial approaches to phenotypic reversion of RDEB and additional human disorders of extracellular matrix, such as junctional EB due to defective laminin 5 , have relied on grafting engineered epidermal tissue (1-3). A number of drawbacks limit applying this approach to humans, including cost, the fragility of engineered epidermal tissue, and the necessity for excising existing tissue prior to grafting, with resultant scarring. Direct administration of viral vectors, on the other hand, suffers from limitations in efficiency, cell targeting, and biosafety. Because they are robust compared with many other cell types, genetically engineered fibroblasts have been explored in a number of applications, including visceral and cutaneous implantation to support bloodstream polypeptide delivery (4-9).

While prior fibroblast-based efforts were encouraging, they often relied on transformed cells and encapsulation or implantation with artificial matrix, thus complicating their practical application. Although epidermal keratinocytes appear to produce the majority of the type VII collagen in the cutaneous basement membrane zone

Received for publication October 21, 2002, and accepted in revised form November 26, 2002.

Address correspondence to: Paul A. Khavari, Program in Epithelial Biology, Stanford University School of Medicine, 269 Campus Drive, Room 2145, Stanford, California 94305, USA Phone: (650) 725-5266; Fax: (650) 723-8762;

E-mail: khavari@CMGM.stanford.edu.

Conflict of interest: The authors have declared that no conflict of interest exists.

Nonstandard abbreviations used: recessive dystrophic

epidermolysis bullosa (RDEB); basement membrane zone (BMZ); overexpressing type VII collagen ( $\mathrm{RDEB}^{+}$cells).
(BMZ), dermal fibroblasts can also contribute to this process (10), raising the possibility that they could serve as delivery vehicles in RDEB. Toward the goal of developing a more straightforward approach to corrective gene delivery, we therefore examined the ability of fibroblasts to express and deliver corrective proteins in RDEB.

\section{Methods}

RDEB cells. Fibroblasts from four unrelated COL7A1 mutant type VII collagen-deficient RDEB patients (3) fulfilling clinical, immunohistological, ultrastructural, and genetic criteria for the disease (11) were grown as described (1). Integrase-based stable integration of the type VII collagen expression plasmid, pCOL7A1, was performed by cotransfecting fibroblasts with a $\phi \mathrm{C} 31$ integrase-encoding plasmid and pCOL7A1 as described (3). For selection, 3 days after transfection, cells were subjected to 10 days of blasticidin $(4 \mu \mathrm{g} / \mathrm{ml})$ in culture media to yield cells overexpressing type VII collagen (RDEB ${ }^{+}$cells). Type VII collagen expression was verified by immunofluorescence microscopy and immunoblot analysis using antibodies to human type VII collagen (CalbiochemNovabiochem Corp., San Diego, California, USA).

Animal studies. For fibroblast injection into mouse skin, 6-week-old athymic nude and CB.17 scid/scid mice were injected intradermally with $10^{6}$ fibroblasts resuspended in $100 \mu \mathrm{l}$ PBS using a 30-gauge needle ( $n=3 \mathrm{mice} /$ cell group). The injection was performed by first piercing the skin, then directing the needle as superficially as possible back upward toward the surface; this commonly led to formation of a well-demarcated papule in the center of the injected area. Eight to 16 weeks after injection, biopsies and analyses were performed on mouse skin. For human skin studies, skin of RDEB patients and normal controls was generated using either early-passage RDEB keratinocytes 
or normal human keratinocytes. Devitalized porcine dermal substrate was used as described (1) because porcine type VII collagen is not detected by the antibodies used in these studies. At least three grafts were regenerated for each of the four patients studied. These grafts were injected with either untreated fibroblasts from RDEB patients (RDEB- fibroblasts deficient in type VII collagen), normal fibroblasts, or RDEB fibroblasts overexpressing type VII collagen (RDEB ${ }^{+}$fibroblasts). Only one of these fibroblast types was injected into each graft, with a minimum of one independent graft injected for each fibroblast group per patient studied. Five normal human skin grafts were also regenerated from patients with normal skin. Two weeks after grafting, $10^{6}$ fibroblasts resuspended in $100 \mu \mathrm{l}$ PBS were injected intradermally into the center of each RDEB graft using a 30-gauge needle as noted above. At 4, 8, and 16 weeks after injection, biopsies and analyses were performed on human skin tissue. All animal studies were conducted using protocols approved by the Stanford Institutional Animal Use Committee.

Analysis of protein expression and tissue ultrastructure. Antibodies to human type VII collagen, including rabbit antisera (Calbiochem-Novabiochem Corp.) and the monoclonal antibody NP185 (a gift of L. Sakai, Shriners Hospital for Children) were used to verify expression of full-length type VII collagen on immunoblot analysis. Cell extracts were prepared, and $50 \mu \mathrm{g}$ of extract protein was electrophoresed on a $6 \%$ polyacrylamide gel. Blots were incubated simultaneously with BRG1, an internal control for protein concentration, extract quality, and transfer efficiency. For immunohistochemistry, skin cryosections were fixed and immunostained with the rabbit antibodies to human type VII collagen followed by a fluoresceinlabeled secondary antibody (Jackson ImmunoResearch Laboratories Inc., West Grove, Pennsylvania, USA). Immunostaining signal was captured by digital microscopy, and type VII collagen signal strength was analyzed in test and normal control samples stained in parallel using optical quantitation software (OpenLab digital imaging package; Improvision Inc., Lexington, Massachusetts, USA). Sections were also analyzed by electron microscopy and immunogold electron microscopy (12) to assess human anchoring fibril formation and ultrastructure.

\section{Results}

Stable gene delivery to primary human fibroblasts. First-passage nontransformed fibroblasts from four previously characterized RDEB patients with COL7A1 mutations that fulfilled all criteria for the disease (11) were isolated and grown as described (13). These cells were engineered for human type VII collagen overexpression. To do this, Streptomyces phage $\phi C 31$ integrase-assisted stable integration $(14,15)$ of the pCOL7A1 plasmid encoding the COL7A1 CDNA driven by the CMV IE promoter was used. This plasmid, generated recently to transfect human keratinocytes (3), was transfected followed by 10 days of blasticidin drug selection enrichment to achieve a stably transfected bulk cell population (3). RDEB fibroblasts stably transfected with pCOL7A1 $\left(\mathrm{RDEB}^{+}\right.$ fibroblasts) express levels of type VII collagen protein that are quantitatively higher even than levels in normal keratinocytes, as opposed to normal fibroblasts, which express significantly lower levels, and unengineered $\left(\mathrm{RDEB}^{-}\right)$fibroblasts, which entirely lack expression (Figure 1a). On a cellular level, $\mathrm{RDEB}^{+}$populations display uniformly detectable type VII collagen protein, in comparison to normal populations which demonstrate lower and more heterogeneous expression (Figure 1b). Engineered fibroblasts overexpressing type VII collagen thus comprise a potential cellular delivery vehicle for RDEB.

Injection of $R D E B^{+}$fibroblasts into mouse skin. To test the ability of $\mathrm{RDEB}^{+}$fibroblasts to deliver type VII collagen to skin, we injected these cells intradermally into intact mouse skin and examined the status of type VII collagen in tissue using human species-specific antibodies. Compared with normal fibroblasts, which produced low levels of type VII collagen centered around murine dermal hair follicles, and $\mathrm{RDEB}^{-}$cells, which yielded no detectable protein, injection of $\mathrm{RDEB}^{+}$cells resulted in correctly localized human type VII collagen at the epidermal-dermal BMZ (Figure 2). Expression was stable for the 16-week duration of the experiment. Type VII collagen overexpression in fibroblasts at higher levels than normal is thus required in this approach because normal cells failed to deliver detectable protein beyond local hair follicles to reach the overlying epidermal BMZ.

Injection of RDEB fibroblasts into RDEB patient skin tissue in vivo. A clinically practical approach to correcting RDEB defects could include direct intradermal injection of human fibroblasts engineered to overexpress type VII collagen. To examine the feasibility of this approach, we injected $\mathrm{RDEB}^{+}$fibroblasts into RDEB human skin tissue regenerated on immune-deficient mice by previously described xenografting methods (16). Both RDEBand normal fibroblasts failed to restore type VII collagen at the cutaneous BMZ or correct subepidermal blistering in any of the injected $\mathrm{RDEB}^{-}$grafts. Injected $\mathrm{RDEB}^{+}$ cells, however, achieved both goals (Figure 3a), with consistent results for the 4-month duration of the experiment in all patient tissue studied. Of interest, border regions more distant from the intradermal injection site displayed blistering in areas transitioning to absent type VII collagen protein (Figure 3a, bottom row), demonstrating the corrective impact of type VII collagen restoration on epidermal-dermal cohesion.

Type VII collagen-positive cells with fibroblast morphology were observed throughout the reticular and papillary dermis of skin tissue injected with $\mathrm{RDEB}^{+}$ fibroblasts at all timepoints (Figure 3b). BMZ-localized type VII collagen was detected up to $75 \mu \mathrm{m}$ away from the nearest type VII collagen-positive cells in the dermis, as verified by serial $5-\mu \mathrm{m}$ cryosections, indicating that this $\mathrm{RDEB}^{+}$fibroblast-produced protein can be delivered from production sites that reside 
a

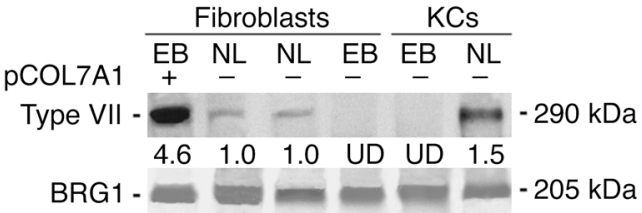

b

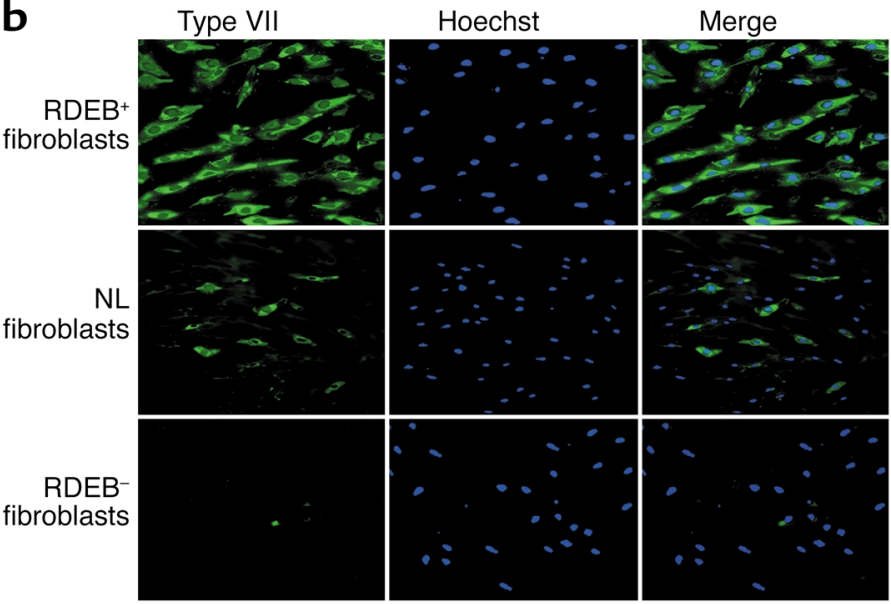

Figure 1

Type VII collagen expression in primary skin cells. (a) Immunoblots of extracts from primary RDEB (EB) and normal (NL) patient skin fibroblasts and keratinocytes (KCs). The first lane represents $\mathrm{RDEB}^{+}$fibroblasts engineered to overexpress type VII collagen. PCOL7A1 is a CMV-driven expression plasmid for type VII collagen (3) stably transfected using the $\phi C 31$ integrase. Optical densitometric quantitation of type VII collagen protein levels are noted below each sample lane, normalized to BRG1 (25), a constitutively expressed control for extract loading, quality, and transfer; normal fibroblasts are assigned a relative value of 1.0. UD, undetectable. (b) Cellular expression of type VII protein expression (green) in engineered $\left(\mathrm{RDEB}^{+}\right)$, normal $(\mathrm{NL})$, and uncorrected (RDEB ${ }^{-}$) fibroblasts. Counterstaining with Hoechst 33342 marks all cellular nuclei (blue). many cell diameters away in the tissue. Detection of BMZ-localized type VII collagen by immunostaining, as assessed by optical signal quantitation software, did not diminish over the course of the experiment compared with normal controls. Combined with the stable retention of type VII collagen-expressing dermal fibroblasts over the timepoints analyzed, this observation suggests persistence of type VII collagen synthesis for the duration of the experiment.

$\mathrm{BMZ}$ anchoring fibrils represent the tangible ultrastructural product formed by type VII collagen (12), and their absence is characteristic of RDEB (17).
Consistent with normalization of correctly localized type VII collagen at the $\mathrm{BMZ}$, injection of $\mathrm{RDEB}^{+}$ fibroblasts restored the presence of anchoring fibrils in RDEB skin (Figure 4), verifying correction of this fundamental ultrastructural disease abnormality.

\section{Discussion}

Injecting genetically engineered fibroblasts offers a number of advantages over the epidermal grafting strategies that have been the mainstay of genodermatoses correction efforts to date $(1,18-20)$, including those recently showing promise for treatment of

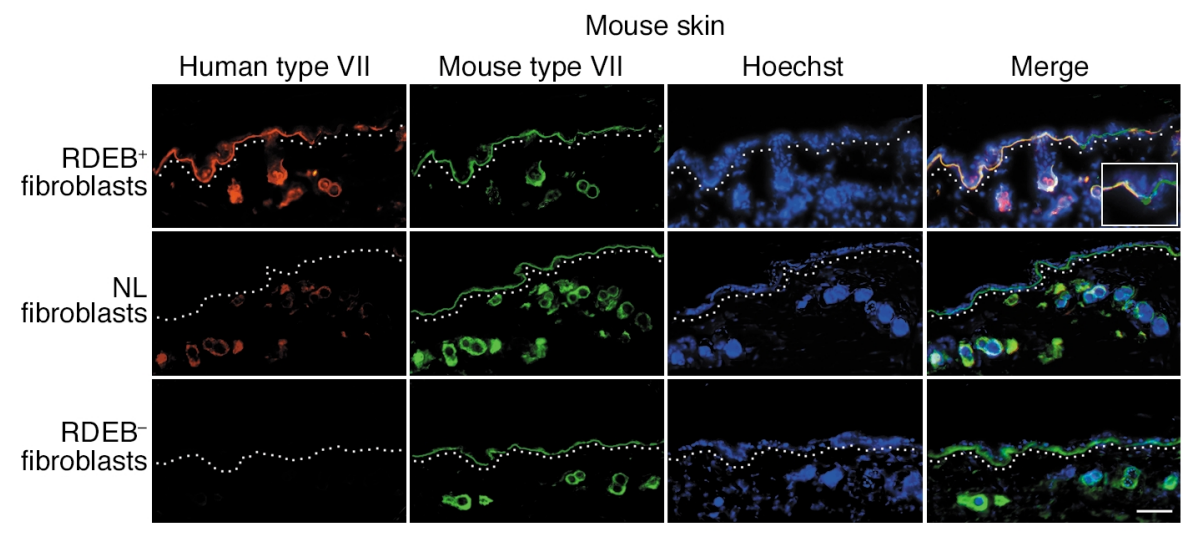

\section{Figure 2}

Intradermal injection of RDEB ${ }^{+}$fibroblasts delivers human type VII collagen to the murine epidermal-dermal junction. Skin from mice injected intradermally with the fibroblast types noted at the left of each row of panels was stained with either the NP185 human-specific monoclonal antibody to type VII collagen (red, first column) or rabbit polyclonal antibodies recognizing both mouse and human type VII collagen (green, second column). Note the presence of human type VII collagen at the cutaneous BMZ in skin injected with RDEB ${ }^{+}$fibroblasts (top row). Note the lack of BMZ-localized type VII collagen in skin injected with normal fibroblasts (middle row) and its complete absence in skin injected with RDEB- fibroblasts (bottom row). Circular structures in the mid to deep dermis are hair follicles; note perifollicular dermal human type VII collagen in dermis injected with RDEB ${ }^{+}$fibroblasts and with the normal fibroblasts (middle row). Merged inset $(\times 40)$ showing skin injected with RDEB+ fibroblasts (top row, far right panel) demonstrates the junction of human type VII collagen detection in the BMZ. Dotted lines denote the upper papillary dermis below the BMZ. Scale bar: $50 \mu \mathrm{m}$. 

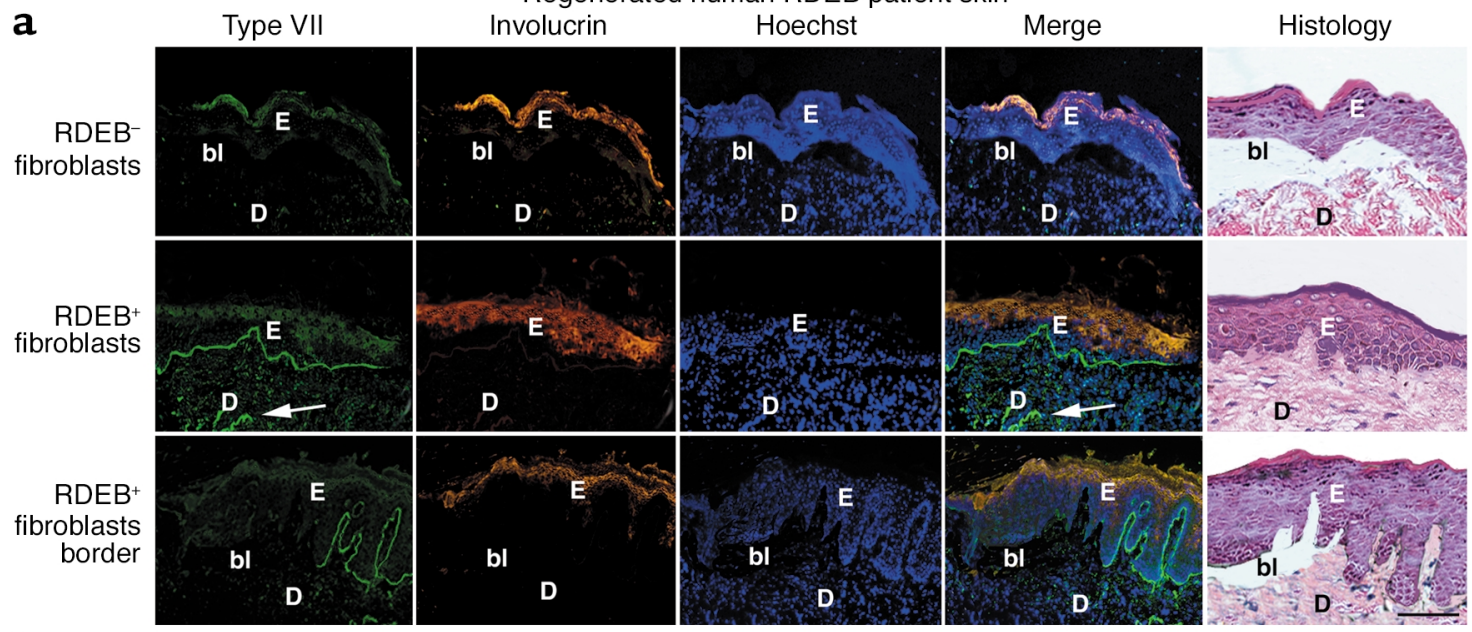

b

RDEB+ fibroblasts
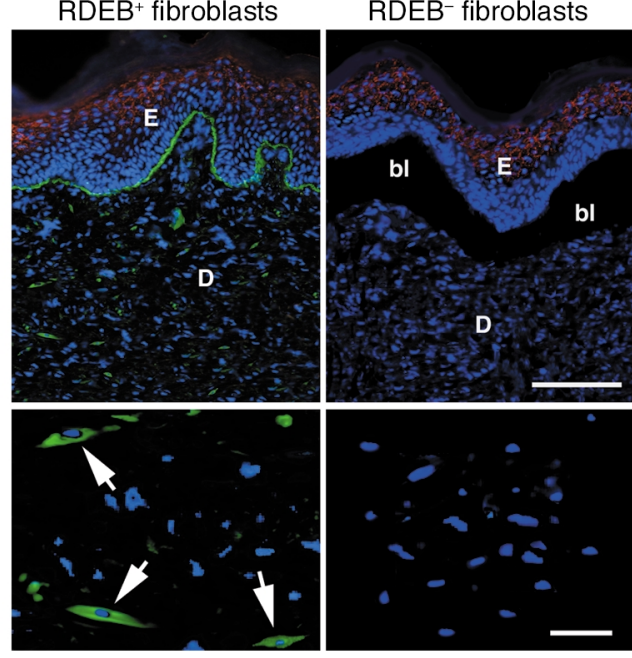

\section{Figure 3}

Fibroblast-mediated type VII collagen delivery to human RDEB skin (a) Type VII collagen (green, first column) is shown in human RDEB skin regenerated on immune-deficient mice after intradermal injection with the cell types noted at left. Note the lack of type VII collagen in skin injected with RDEB- fibroblasts (top row) and its BMZ localization in skin injected with $\mathrm{RDEB}^{+}$fibroblasts (middle row; arrows in dermis denote a collection of intradermal $\mathrm{RDEB}^{+}$cells). Note the blistering seen past the border of human type VII collagen protein (bottom row). The human origin of the skin tissue studied was confirmed using species-specific antibodies to involucrin (orange, second column); triple-stained specimens with serial histological sections are shown. Scale bar: $50 \mu \mathrm{m}$. (b) RDEB fibroblasts in RDEB skin tissue. Note type VII collagen-positive dermal cells (arrows) with typical elongated fibroblast morphology in tissue injected with $\mathrm{RDEB}^{+}$cells and the complete absence of detectable type VII collagen in RDEB skin tissue injected with RDEB ${ }^{-}$cells. Scale bars: top panels, $75 \mu \mathrm{m}$; bottom panels, 15 $\mu \mathrm{m}$. E, epidermis; D, dermis; bl, blister.
$\operatorname{RDEB}(2,3)$. Fibroblasts are more robust cells than keratinocytes, and are less susceptible to growth arrest and differentiation than epidermal progenitors. Unlike epidermal sheets, which are fragile and must be used immediately, fibroblasts can be frozen, with partitioned stocks available for growth and readministration to the patient. Moreover, in contrast to epidermal cells, which require wounding for successful engraftment, purified fibroblasts can be delivered to intact skin via intradermal injection, eliminating the need for special subsequent wound care. This is especially important in generalized disorders such as RDEB, where corrective epidermal grafting could require removing most of the epidermis. Intradermal injection of genetically engineered keratinocytes can lead to undesirable cyst formation (21), and we have observed that injected human keratinocytes fail to persist in dermal tissue (22). Finally, the approach described here is simplified in that it relies on injected cells alone and has no requirement for cell encapsulation or delivery within a coadministered matrix.

The major disease features of RDEB were corrected by injecting genetically engineered fibroblasts into regenerated RDEB skin tissue on scid/scid mice. This correction required overexpression of type VII collagen, as normal fibroblasts failed to deliver detectable protein to the BMZ. Nonviral approaches to such overexpression of secreted therapeutic proteins include the $\phi C 31$ integrase approach used here as well as the Sleeping Beauty transposase $(23,24)$. Defining the potential advantages and drawbacks of these plasmid integration strategies compared with conventional approaches that

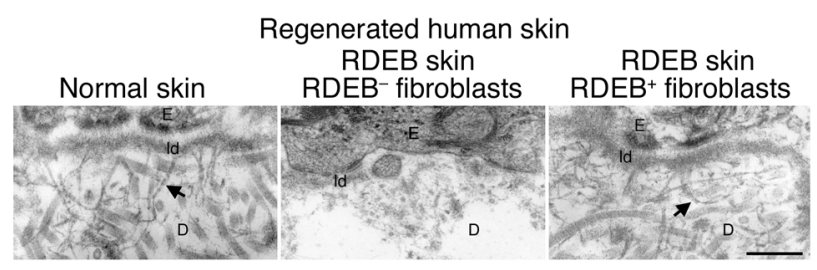

\section{Figure 4}

Restoration of anchoring fibrils after intradermal fibroblast injection. $\mathrm{BMZ}$ ultrastructure of human skin tissue regenerated on CB.17 scid/scid mice. Note the absence of anchoring fibrils in RDEB skin injected with $\mathrm{RDEB}^{-}$fibroblasts (middle panel) and their restoration in RDEB skin injected with $\mathrm{RDEB}^{+}$fibroblasts (right panel). Normal skin control (left panel) was produced using cells from normal subjects. Arrows denote representative anchoring fibrils. Scale bar: $500 \mathrm{~nm}$. Id, lamina densa. 
use electroporation and drug selection (8) is of significant interest for future study. Injection of genetically engineered fibroblasts thus holds promise in correcting human disorders of cutaneous and visceral tissues characterized by defects in secreted matrix proteins.

\section{Acknowledgments}

This work was supported by the United States Veteran Affairs Office of Research and Development, by NIH grant AR-44012 (to P.A. Khavari), and by the Epidermolysis Bullosa Medical Research Foundation. S. Ortiz-Urda was supported by the Max Kade Foundation. We thank K. Wolff and K. Rappersberger for support, M. Calos and B. Thyagarajan for helpful discussions, J. Vogel and M. Fang for COL7A1 sequences, L. Nall for assistance with patient tissue, S. Tufa for technical assistance, and P.B. Robbins, F. Scholl, Z. Siprashvili, A. Nguyen, N. Griffiths, and P. Bernstein for technical advice, support, and helpful discussions.

1. Robbins, P.B., et al. 2001. In vivo restoration of laminin 5 beta 3 expression and function in junctional epidermolysis bullosa. Proc. Natl. Acad. Sci. USA. 98:5193-5198.

2. Chen, M., et al. 2002. Restoration of type VII collagen expression and function in dystrophic epidermolysis bullosa. Nat. Genet. 32:670-675.

3. Ortiz-Urda, S., et al. 2002. Stable nonviral genetic correction of inherited human skin disease. Nat. Med. 8:1166-1170.

4. Petersen, M.J., et al. 1995. Sustained production of human transferrin by transduced fibroblasts implanted into athymic mice: a model for somatic gene therapy. J. Invest. Dermatol. 104:171-176.

5. Garver, R.I., Jr., Chytil, A., Courtney, M., and Crystal, R.G. 1987. Clonal gene therapy: in vivo expression of a transplanted monoclonal population of murine fibroblasts containing a retrovirus inserted human alpha 1-antitrypsin gene. Trans. Assoc. Am. Physicians. 100:10-20.

6. Scharfmann, R., Axelrod, J.H., and Verma, I.M. 1991. Long-term in vivo expression of retrovirus-mediated gene transfer in mouse fibroblast implants. Proc. Natl. Acad. Sci. USA. 88:4626-4630.

7. Serguera, C., Bohl, D., Rolland, E., Prevost, P., and Heard, J.M. 1999. Control of erythropoietin secretion by doxycycline or mifepristone in mice bearing polymer-encapsulated engineered cells. Hum. Gene Ther. 10:375-383.

8. Roth, D.A., Tawa, N.E., Jr., O’Brien, J.M., Treco, D.A., and Selden, R.F.
2001. Nonviral transfer of the gene encoding coagulation factor VIII in patients with severe hemophilia A. N. Engl. J. Med. 344:1735-1742.

9. Krueger, G.G. 2000. Fibroblasts and dermal gene therapy: a minireview. Hum. Gene Ther. 11:2289-2296.

10. Marinkovich, M.P., Keene, D.R., Rimberg, C.S., and Burgeson, R.E. 1993. Cellular origin of the dermal-epidermal basement membrane. Dev. Dyn. 197:255-267.

11. Marinkovich, M.P., Herron, G.S., Khavari, P.A., and Bauer, E.A. 1999. Hereditary epidermolysis bullosa. In Dermatology in general medicine. Volume 1. Fifth edition. I.M. Freedberg et al., editors. McGraw-Hill Health Professions Division. New York, New York, USA. 690-702.

12. Keene, D.R., Sakai, L.Y., Lunstrum, G.P., Morris, N.P., and Burgeson, R.E. 1987. Type VII collagen forms an extended network of anchoring fibrils. J. Cell Biol. 104:611-621.

13. Freiberg, R.A., Ho, S.N., and Khavari, P.A. 1997. Transcriptional control in keratinocytes and fibroblasts using synthetic ligands. J. Clin. Invest. 99:2610-2615.

14. Kuhstoss, S., and Rao, R.N. 1991. Analysis of the integration function of the streptomycete bacteriophage phi C31. J. Mol. Biol. 222:897-908.

15. Thyagarajan, B., Olivares, E.C., Hollis, R.P., Ginsburg, D.S., and Calos, M.P. 2001. Site-specific genomic integration in mammalian cells mediated by phage phiC31 integrase. Mol. Cell. Biol. 21:3926-3934.

16. Kim, Y.H., Woodley, D.T., Wynn, K.C., Giomi, W., and Bauer, E.A. 1992. Recessive dystrophic epidermolysis bullosa phenotype is preserved in xenografts using SCID mice: development of an experimental in vivo model. J. Invest. Dermatol. 98:191-197.

17. Bruckner-Tuderman, L., Hopfner, B., and Hammami-Hauasli, N. 1999. Biology of anchoring fibrils: lessons from dystrophic epidermolysis bullosa. Matrix Biol. 18:43-54.

18. Khavari, P.A., Rollman, O., and Vahlquist, A. 2002. Cutaneous gene transfer for skin and systemic diseases. J. Intern. Med. 252:1-10.

19. Choate, K.A., Medalie, D.A., Morgan, J.R., and Khavari, P.A. 1996. Corrective gene transfer in the human skin disorder lamellar ichthyosis. Nat. Med. 2:1263-1267.

20. Freiberg, R.A., et al. 1997. A model of corrective gene transfer in X-linked ichthyosis. Hum. Mol. Genet. 6:927-933.

21. Gagnoux-Palacios, L., et al. 1996. Functional re-expression of laminin-5 in laminin-gamma2-deficient human keratinocytes modifies cell morphology, motility, and adhesion. J. Biol. Chem. 271:18437-18444.

22. Lazarov, M., et al. 2002. CDK4 coexpression with Ras generates malignant human epidermal tumorigenesis. Nat. Med. 8:1105-1114.

23. Yant, S.R., et al. 2000. Somatic integration and long-term transgene expression in normal and haemophilic mice using a DNA transposon system. Nat. Genet. 25:35-41.

24. Olivares, E.C., et al. 2002. Site-specific genomic integration produces therapeutic factor IX levels in mice. Nat. Biotechol. 20:1124-1128.

25. Khavari, P.A., Peterson, C.L., Tamkun, J.W., Mendel, D.B., and Crabtree, G.R. 1993. BRG1 contains a conserved domain of the SWI2/SNF2 family necessary for normal mitotic growth and transcription. Nature. 366:170-174 